\title{
Sciences and audiences along the last century: the impact of Astronomy Education
}

\author{
Rosa M. Ros ${ }^{1}$ and Beatriz García ${ }^{2}$ \\ ${ }^{1}$ Unversidad Politécnica de Cataluña, Spain. email: rosamariaros27@gmail.com \\ ${ }^{2}$ ITeDAM-CONICET-CNEA-UNSAM, UTN Mendoza, Lab. Pierre Auger
}

\begin{abstract}
Just as in the past, the development of the natural sciences and in particular of astronomy has changed the history of humanity. If we think about the role of our discipline into the future, it shows its enormous power in the field of education, owing to the possibility of awakening interest in science in very varied audiences. Within the framework of the enormous progress made in the technologies related to astronomy, many of them of daily use, the role of the astronomer in the era of Communications acquires fundamental importance.

In this presentation, we will try to make a journey through the different ways of presenting astronomical topics for different audiences over the last 100 years. In turn, we will show some specific achievements, associated with education programmes of the discipline. We discuss the impact produced by proposals that are both rigorous in terms of content, and also appeal to the development of the human being in an integral manner, within the framework of citizen science activities.

For this research, we have taken into account the uninterrupted development of the NASE programme, which has performed 112 courses in 24 countries throughout the world and in different languages. NASE has involved 4966 secondary teachers in the last eight years.
\end{abstract}

Keywords. Education in astronomy, NASE programme

\section{Past and Present of Science Education and Astronomy impact}

Education, and in particular astronomy teaching, changed a lot in a century. One hundred years ago the people that arrived at secondary school were much more motivated and interested than now. Of course, at that time, only a reduced number of young people studied at secondary level, and even fewer did so at university. In addition, society had different values than now. The sacrifice spirit and discipline helped teachers to present sciences with less difficulty than now. At present, and in some countries, many people go to secondary schools. When the number of students increases, normally the motivation and level decrease. The majority of students in the secondary school do not have a clear idea about how or why they should continue their studies in a university. This big group of students dislike sciences in general, maybe because they do not assimilate information very well. They believe that science is boring and difficult to follow in the classroom. Now, the teachers need to introduce mathematics, physics and other science matters from a more attractive approach. Science needs to be interesting and challenging, but this is not easy to achieve (Fig. 1). Astronomy could be the solution!

From the beginning, humanity felt a special relation with astronomy. Teenagers feel this special relationship with astronomy too. It is difficult to know the reason, but it is easy to observe their interest. Really, astronomy is a special science. Astronomy is the only science that has a very big number of astronomy amateurs, who work strongly (outside their periods of work) in order to increase their knowledge about a specific 

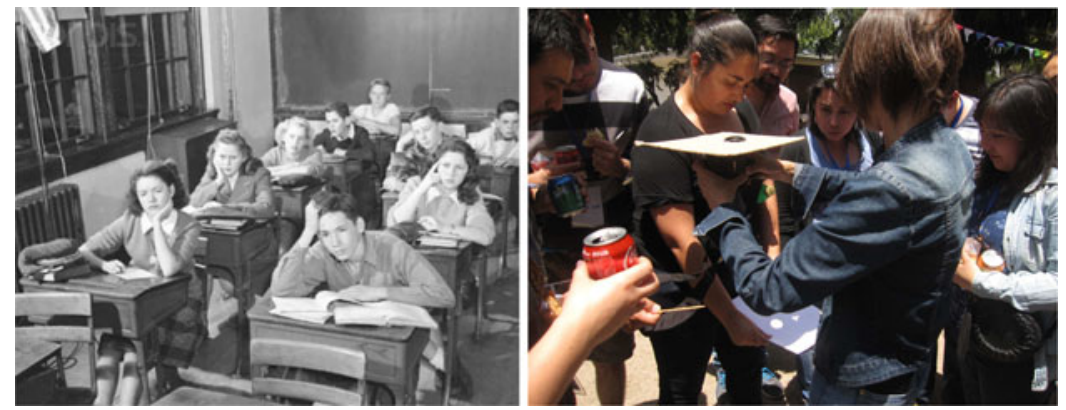

Figure 1. Challenges in education: the past (left) and now (right).

astronomical topic. They spend nights and nights making observations, taking photos and communicating their results to other colleagues. Of course, it is a good idea that each secondary school should try to make contact with an amateurs astronomical association, in order to have support preparing astronomical observations for students.

Teenagers are interested in astronomy, and we can use this opportunity in order to introduce science by means of astronomy in a much more motivating way. Physics is the same here on Earth as in the Universe, but it is more suggestive to learn about stellar evolution, how stars are born, live and die, than to study physics in a purely classical way. A mathematics teacher may have difficulties to motivate his students to solve a right-angled triangle, when two angles and a side are known, but it is easy to interest students to calculate the distance from the Earth to the Sun or to the Moon.

Astronomy has another set of characteristics that are not so common in other sciences. Astronomy is a science that make predictions. Using orbital calculations, it is possible to predict eclipses, transits and other phenomena. Of course, predictions have an interest for humanity, and in some ways this aspect was not so good for astronomy, because that some people in the past believed that it was possible to make predictions about their lives, and hence astrology. appeared on the scene. Of course, science teachers could explain the difference between astrology and astronomy. This is also interesting to students.

The other characteristic that we should mention is that astronomy is a very visible science pictorially. There are a lot of wonderful pictures in relation to astronomy our senses naturally fall in love with. Professional telescopes obtain some of these images and they are very spectacular. We can see others with an amateur telescope, or in the school yard. For instance, how can one forget the first time that you observe the Moon's surface. It is not comparable with a film or a picture. Almost every year we can enjoy a lunar eclipse, and this is a good occasion to introduce astronomy too. In any case, the main idea is that the student feels that he/she is the main actor in the learning process. The approach is easier because "astronomy is visible and they can make simple observations in the school yard by themselves. Astronomy is motivating for them, maybe because it makes predictions, or simply because astronomy was always interesting for humanity. Students want to know more about exoplanets and new worlds, of course.

In NASE courses, all these ideas are inspiring the participant teachers. During the course, the participants learn to observe, to prepare some simple devices and to do it because all the schools have a sky over them, and we promote this to be used as an astronomy laboratory. In NASE workshops, we use photographs made by professional observatories and also amateurs. In some of them, we show several activities that could be used also in mathematics, physics and chemistry classes. We help to change science 

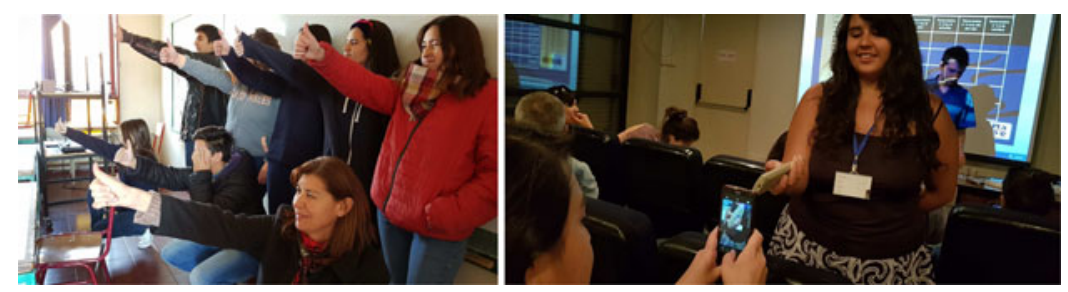

Figure 2. Understanding parallax (left); detecting the infrared (right).

classes into active and interesting classes, where the students are the main actors in their learning process. If they enjoy learning, they generally do it well.

\section{Astronomy as a door to Science}

In our society astronomy is well regarded for students and people in general. Astronomy continues to be a part of ancestral knowledge, that humanity feels they are part of the wider unknown, including from the mystery and philosophy points of view. This interest continues as the future development of all of us, with the result that young people and teenager students often feel that they have several questions about the wider universe. This curiosity is a good opportunity to introduce science into the pedagogic process, which in general is not necessarily so suggestive to all of them. This situation is more and more explored now, because astronomy offers an observational approach.

In the past, astronomy was presented in a more theoretical way than it is now. At present we have the opportunity to showcase astronomy as a practical activity, making observations in situ or with robotic telescopes. Space science also increases its presence in our lives, and students can feel its call. But one of the most important characteristics of astronomy is the excellent colour pictures that new technologies offer to us. This aspect gives many opportunities to communicate using the media. And really astronomy is more and more present on TV and in the newspapers. Of course, it is easier to motivate interest with an enormous colour photo than a small black and white one. Astronomy is also present by means of applications in our lives, such as 3D-movies using the parallax concept, or something in astrophysics (Fig. 2).

\section{Learning by doing in Astronomy}

A hundred years ago, astronomy education was open to a limited number of interested people, and its approach was more theoretical than other sciences. When education was open to many more people, fifty years ago, it was necessary to sell the product much better, and it was necessary to introduce an approach that was more motivating. In general, education changed in many aspects and began to take on a much more active presentations.

The advantage of astronomy over other sciences is that all schools have an astronomy lab, that is to say the sky over the school yard. This is an important facility in order to offer a methodology of learning by doing. Secondary schools often do not have advanced astronomical devices to make observations, but we can always promote that students construct for themselves simple devices, such as quadrants (Fig. 3) or goniometers, and even telescopes if they have a good teacher.

This characteristic of astronomy is that it is accessible to everybody, and in particular, astronomy is a special science for a lot of amateurs. They are motivated and enjoy working strongly in the study and observation of the sky, and in some cases, by making telescopes and other devices themselves. 


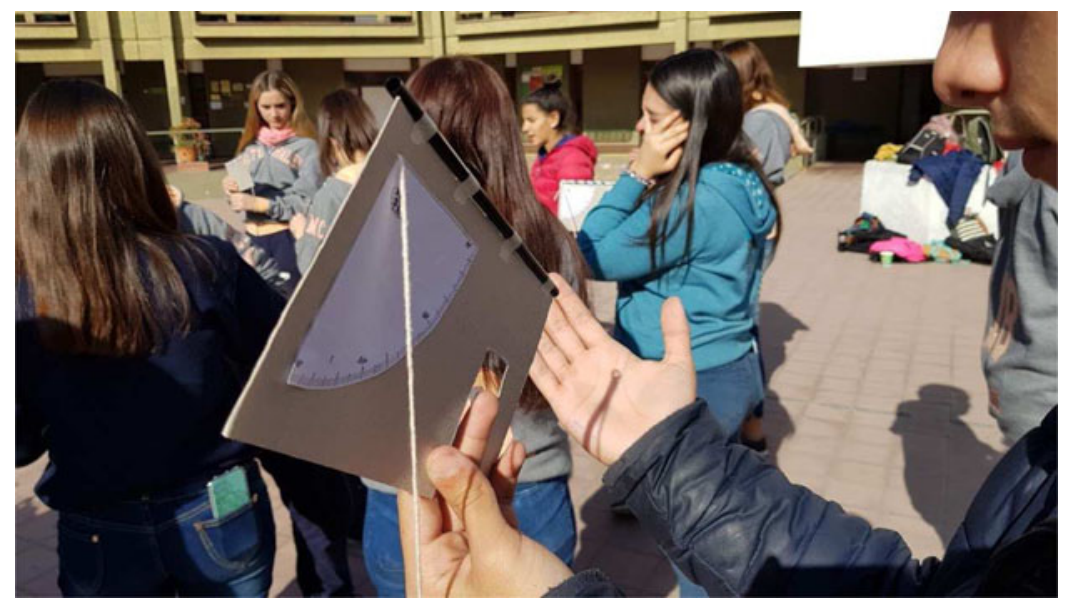

Figure 3. A student using a quadrant made of simple materials, to measure the Sun's altitude.

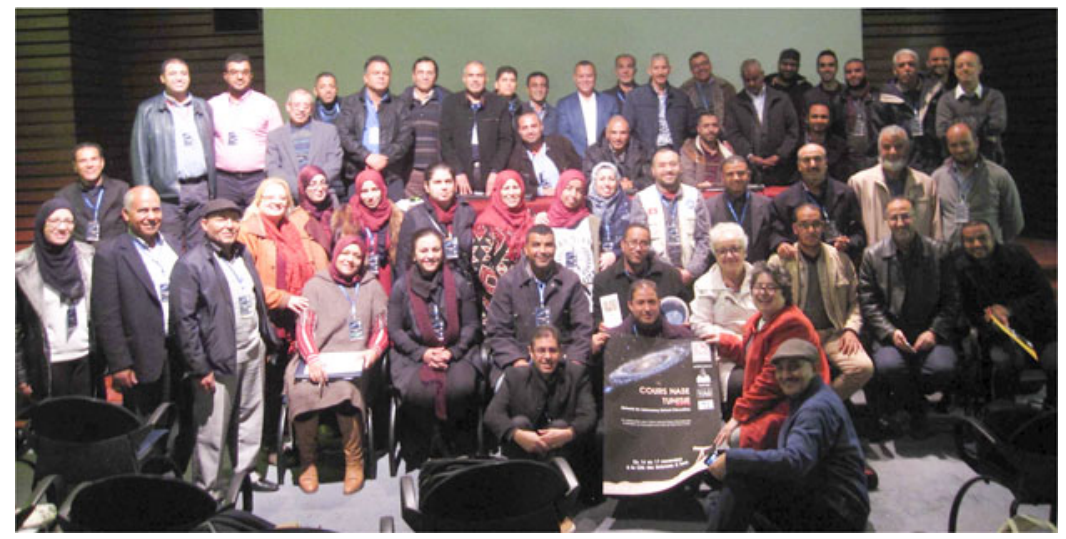

Figure 4. NASE course in Tunisia, 2018.

\section{The NASE Programme}

The NASE Working Group was created at the IAU GA in Rio de Janeiro in 2009. During the International Year of Astronomy in 2009 we organized a couple of pilot courses in Ecuador and Peru in cooperation with UNESCO. This experience was a basis for our materials, conferences and workshops, which we presented in the NASE textbook "14 steps to the Universe" (Ros 2018). Currently NASE has organized 112 courses in 24 countries, involving about 5000 teachers from all over the Globe in different languages in the last eight years. One of the NASE groups is shown in Fig. 4, a very successful meeting in Tunisia on 2018. In Argentina NASE obtained the ISO 29990:2010 certification, in the frame of the Learning services for non-formal education and training - Basic requirements for service providers (ISO 2014)(ISO 2017).

\section{IAU Decanal Plan 2010-2020: NASE was born}

George Miley prepared a decadal plan for the IAU. In this plan, the necessity of increasing the IAU presence in pre-university education was stressed. In order to train more astronomers, it was necessary to show students before they enter university what 
astronomy was really about. It was specially necessary to introduce astronomy at secondary school, and the best way was by means of its teachers. It was evident that the role of astronomy in secondary schools was needed throughout the whole world.

In an earlier meeting in 2008 at the Paris Observatory, the basic idea of a new school for secondary school teachers was mooted and the first ideas about a new Working Group for the training of astronomy teachers appeared. Thus was born NASE (Network for Astronomy School Education). NASE's main purpose is the development of highlevel teacher professional development in all countries that are interested in astronomy education at different levels, incorporating issues related to the discipline in different curriculum areas, with the aim of introducing young people to science through the study of the universe.

This proposal, the initial teacher training in astronomy with conferences, workshops, working groups, visits to the city and archeological areas with astronomical themes (whenever this is possible) is a new way of education, where students and teachers converge to reach a better knowledge of nature by means of observation, and "hands on" activities and the reflecting on their achievements.

\section{Acknowledgements}

The authors want to thanks the IAU, the Spanish National Research Council (CSIC), the Technical University of Catalonia, the Argentinean National Council of Scientific Research (CONICET) and the National Technical University-Mendoza for supporting this work.

\section{References}

Belmonte, J. A., Berthomieu, F., da Costa, A., Deeg, H., Deustua, S., Fiero, J., García, B., Hemenway, M.K., Moreno, R., Pasachoff, J., Percy, J., Ros, R., Stavinschi, M. 2018, 14 steps to the Universe, NASE publications, Albedo ed., Barcelona

Cano, E. and Bartolomé, A. (2014). Evaluar la formación es posible. Avaliar a formação é possivel, Col-lecció Transmedia XXI. Laboratori de Mitjans Interactius, Barcelona

ISO (2014), Quality assurance in adult learning - Implementation Guide of Quality Management Systems (ISO 9001, ISO 29990), QUADRANT Projet, www.quadrat-project.eu/index. $\mathrm{php} / \mathrm{results} / \mathrm{handbook}$

ISO (2017), ISO 29990:2010(en) Learning services for non-formal education and training. Basic requirements for service providers, www.iso.org/obp/ui/iso:std:iso:29990:ed-1:v1:en 